\title{
Discrete-Time State Dependent Bulk Service Queue with Multiple Vacations and Changeover Times
}

\author{
P. Vijaya Laxmi and D. Seleshi \\ Department of Applied Mathematics, Andhra University, Visakhaptnam 530003, India \\ Correspondence should be addressed to P. Vijaya Laxmi; vijaya_iit2003@yahoo.co.in
}

Received 1 February 2014; Accepted 24 February 2014; Published 24 March 2014

Academic Editors: R. Varela and X.-M. Yuan

Copyright (C) 2014 P. Vijaya Laxmi and D. Seleshi. This is an open access article distributed under the Creative Commons Attribution License, which permits unrestricted use, distribution, and reproduction in any medium, provided the original work is properly cited.

\begin{abstract}
This paper presents the analysis of a discrete-time renewal input multiple vacations queue with state dependent service and changeover times under $(a, c, b)$ policy. The service times, vacation times, and changeover times are geometrically distributed. The server begins service if there are at least $c$ units in the queue and the services are performed in batches of minimum size $a$ and maximum size $b(a \leq c \leq b)$. At service completion instant, if the queue size is less than $c$ but not less than a secondary limit $a$, the server continues to serve and takes vacation if the queue size is less than $a-1$. The server is in changeover period whenever the queue size is $a-1$ at service completion instant and $c-1$ at vacation completion instant. Employing the supplementary variable and recursive techniques, we have derived the steady state queue length distributions at prearrival and arbitrary epochs. Based on the queue length distributions, some performance measures of the system have been discussed. A cost model has been formulated and optimum values of the service and vacation rates have been evaluated using genetic algorithm. Numerical results showing the effect of model parameters on the key performance measures are presented.
\end{abstract}

\section{Introduction}

The interest in discrete-time queues in which time is slotted (divided into fixed-length of contiguous intervals) has got a spectacular growth with the arrival of the digital technologies. A fundamental motive to study discrete-time queues is that they are more appropriate than their continuous-time counterparts for analyzing computer and telecommunication systems, since nowadays these systems are more digital (such as a machine cycle time, bits, and packets) than analogical. In view of this they have become increasingly important due to their applications in the study of many computer and communication systems such as asynchronous transfer mode (ATM) multiplexers, broadband integrated services digital network (B-ISDN), circuit-switched time-division multiple access (TDMA) systems, slotted carrier-sense multiple access (CSMA) protocols, and traffic concentrators in which the time axis is divided into slots. Further, the advantage of analyzing a discrete-time queue is that one can obtain the continuous-time results from it as a limiting case but the converse is not true. However, from an applied and a theoretical point of view both discrete and continuous-time queueing models have importance. One can see Bruneel and Kim [1], Takagi [2], Tran-Gia et al. [3], and Robertazzi [4] for extensive treatments of various types of discrete-time queues and their applications.

Batch service queues have potential applications in telecommunication systems, service mechanisms of a web server, and computer operating systems. In addition to these they also have applications in production, manufacturing, and traffic signal systems where the processor processes packets in batches without overloading the system. In such batch service systems, jobs arriving one at a time must wait in the queue until a sufficient number of jobs get accumulated. Studies on the queueing systems with batches have been reported in Medhi [5] and Chaudhry and Templeton [6]. Gupta and Goswami [7] discussed analytic and computational aspects of Geo/ $G^{a, b} / 1 / N$ queue. Chaudhry and Chang 
[8] considered a discrete-time bulk service Geo $/ G^{Y} / 1 / N+B$ queue. They discussed both the analytic and computational aspects of the distributions of the number of customers in the queue at postdeparture, random, and prearrival epochs. Goswami et al. [9] analyzed discrete-time single server infinite (finite) buffer bulk service queues. The interarrival time of successive arrivals and service times of batches are assumed to be independent and geometrically distributed.

Batch service queues with server vacations have become more effective in modeling and analyzing communication networks and other engineering systems. Such models are useful for the systems in which the server wants to utilize the idle time for different purposes. The discrete-time GI/Geo/1 queue with multiple vacations under late arrival system with delayed access (LAS-DA) has been studied by Tian and Zhang [10]. By using matrix-geometric method, they obtained explicit expressions for the stationary distributions of queue length and waiting time. Goswami and Vijaya Laxmi [11] analyzed a discrete-time finite buffer single server accessible and nonaccessible batch service queue with multiple vacations. The interarrival times of customers are assumed to be arbitrarily distributed. The service times and vacation times are assumed to be geometrically distributed.

In many real-life queueing situations, jobs are served with a control limit policy. For example, in some manufacturing systems it is possible to process jobs only when the number of units to be processed exceeds a specified level and when service starts it is profitable to continue it even when the queue size is less than the specified level but not less than a secondary limit. Tadj and Abid [12] discussed optimal management policy for a single server and single or bulk service characterized by the bilevel service discipline. Baburaj [13] presented a discrete time bulk service queue under the $(a, c, d)$ policy. The interarrival and service times are assumed to be geometrically distributed.

Queues with single server whose service and vacation rates depend on the queue size are realistic models for systems whose server's speed needs to be adjusted according to the number of customers waiting in line. Chao and Rahman [14] analyzed a finite buffer renewal input queue with state dependent services and vacations. They have computed the queue length distributions using supplementary variable and recursive techniques. Parthasarathy and Sudhesh [15] considered a time-dependent single server retrial queue with state dependent rates. They have obtained the time-dependent system size probabilities by employing continued fractions analytically in closed form in terms of convolutions.

The above literature survey reveals that much work has not been focused on discrete-time renewal input multiple vacation queues with changeover times and state dependent services. In most of the literature presented before, it is assumed that the time taken by the server to change the state (from regular working state to vacation state and vice versa) is negligible. However, in many real life situations, the server takes some time to switch from regular working period to vacation period and vice versa. IEEE 802.16-2009 is one such protocol which has three types of power saving classes (types I, II, and III) based on sleep mode operations. Type III sleep mode operations justify our model, wherein the sleep mode and sleep delay mode correspond to vacations and changeover times, respectively. Therefore, the study of queues with vacations and changeover times is important for the design and implementation of communication networks. We aim to introduce state dependent services and changeover times to discrete-time renewal input queue with multiple vacations. One may note that the general uncorrelated arrival process appears to be more appropriate and reasonable than the geometric (Geo) distribution and also it can include the special cases of geometric, deterministic (Det), and arbitrary (Arb) distributions. Further, we also carry out cost analysis as cost is an important aspect that leads to the economic interpretation of the queueing system. Optimization techniques are widely used to effectively assess the performance of the systems. One can construct a suitable cost model and determine the optimum values of the parameters using various optimization techniques. We have employed genetic algorithm (GA) for this purpose. Genetic algorithm works by mimicking the evolutionary principles and chromosomal processing in natural genetics. GA's intelligent search procedure finds the best and fittest design solutions, which are otherwise difficult to find using other optimization techniques. The method was developed by Holland [16]. For the advantages of GA one may refer to R. L. Haupt and S. E. Haupt [17] and Rao [18].

This paper focuses on an infinite buffer discrete-time state dependent bulk service queue with multiple vacations and changeover times under $(a, c, b)$ policy. The interarrival times are assumed to be arbitrarily distributed. The service time of batches, vacation, and changeover times are assumed to be geometrically distributed. We provide a recursive method using the supplementary variable technique to develop the steady state queue length distributions at various epochs for LAS-DA system.

The rest of the paper is organized as follows. Section 2 presents the description of the model and some notations used in this paper. Section 3 deals with the steady state distributions at prearrival and arbitrary epochs including some results that can be deduced from our model by taking specific values of some of the parameters. Performance measures of the system and cost model are presented in Section 4. Using some numerical results, we demonstrate the parameter effect on the performance measures of the system in Section 5. Finally, Section 6 concludes the paper.

\section{The Model Description}

Let us consider a discrete-time infinite buffer bulk service queue with multiple vacations (MV) and changeover times under LAS-DA. The interarrival and service times of customers are assumed to be arbitrarily and geometrically distributed, respectively. The interarrival times $A$ of two successive arrivals are independent and identically distributed (i.i.d.) random variables (r.v.s) with common probability mass function (p.m.f.) $a_{i}=P(A=i), i \geq 1$, corresponding probability generating function (p.g.f.) $A(z)=\sum_{i=1}^{\infty} a_{i} z^{i}$, and mean interarrival time $1 / \lambda=A^{(1)}(1)$, where $h^{(1)}\left(x_{0}\right)$ is the first derivative of $h(x)$ with respect to $x$ at $x=x_{0}$. 
The service times $S$ of batches are independent and geometrically distributed with common (p.m.f.) $P(S=i)=\bar{\mu}_{j}^{i-1} \mu_{j}$, $0<\mu_{j}<1, i \geq 1, j=1,2$ and mean service time $1 / \mu_{j}$, where for any real number $x \in[0,1]$, we denote $\bar{x}=1-x$. The service begins only if there are at least $c$ units in the queue. The customers are served in batches of minimum size $a$ and maximum size $b(a \leq c \leq b)$ with service rate depending on the queue size $n$ as follows: if the queue size is less than $a$, the batch under consideration is served with rate $\mu_{1}$ and if the queue size is greater than $a$, the batch is served with rate $\mu_{2}$. Further, we assume that the service rate depends only on the queue size but on the batch size because the batches considered for service are of variable sizes with minimum $a$ and maximum $b$.

At a service completion epoch (i) if the queue size is less than $c$ but not less than $a$ then the server continues to serve, (ii) if there are $j$ customers $(0 \leq j \leq a-2)$ in the queue then the server will go for $\mathrm{MV}$, and (iii) if the queue size is $a-1$ then instead of going for vacation immediately the server waits for some time in the system called changeover time which is geometrically distributed with rate $\alpha_{1}$. It starts service on finding an arrival during this changeover time; otherwise, it will go for vacation. The vacation times are assumed to be independent and geometrically distributed with rate $\phi$.

On returning from a vacation (i) if the server finds $j$ customers $(0 \leq j \leq c-2)$ in the queue, it takes another vacation and so on until at least $c-1$ customers are available in the queue, (ii) if there are $c-1$ customers in the queue, the server is in changeover time, which is again geometrically distributed with rate $\alpha_{2}$. It starts service on finding an arrival during this changeover time; otherwise, another vacation follows, and (iii) if the server finds $j \geq c$ customers in the queue, $\min \{j, b\}$ customers are taken for service according to FCFS discipline. The traffic intensity is given by $\rho=2 \lambda / b\left(\mu_{1}+\right.$ $\left.\mu_{2}\right)$.

Let the time axis be slotted into intervals of equal length with the length of a slot being unity. Further, let the time axis be marked as $0,1,2, \ldots, t, \ldots$ and assume that a potential arrival occurs in the interval $(t-, t)$ and a potential departure occurs in the interval $(t, t+)$ as shown in Figure 1.

The state of the system prior to a potential arrival (at $t-$ ) is described by the following (r.v.s):

(i) $N_{q}(t-)=$ number of customers present in the queue (excluding the batch in service);

(ii) $U(t-)=$ the remaining interarrival time for the next arrival;

(iii)

$\xi(t-)= \begin{cases}0, & \text { if the server is on vacation, } \\ 1, & \text { if the server is busy, } \\ 2, & \text { if the server is in changeover time. }\end{cases}$

Let us define the following joint probabilities:

$$
\begin{array}{r}
P_{n, 0}(u, t-)=P\left\{N_{q}(t-)=n, U(t-)=u, \xi(t-)=0\right\}, \\
n \geq 0, \quad u \geq 0,
\end{array}
$$

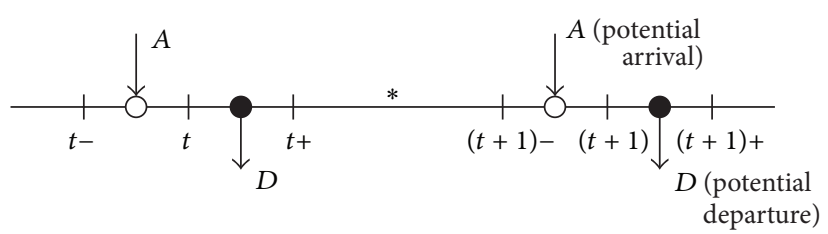

O Potential arrival epoch $\quad(t+,(t+1)-)$ Outside observer's interval

- Potential departure epoch $t+$ Epoch after a potential departure

* Outside observer's epoch $t$ - Epoch prior to a potential arrival

FIGURE 1: Various time epochs in LAS-DA.

$$
\begin{array}{r}
P_{n, 1}(u, t-)=P\left\{N_{q}(t-)=n, U(t-)=u, \xi(t-)=1\right\}, \\
n \geq 0, \quad u \geq 0, \\
P_{n, 2}(u, t-)=P\left\{N_{q}(t-)=n, U(t-)=u, \xi(t-)=2\right\}, \\
n=a-1, c-1, \quad u \geq 0 .
\end{array}
$$

At steady state, let

$$
\begin{gathered}
P_{n, 0}(u)=\lim _{t \rightarrow \infty} P_{n, 0}(u, t-), \quad n \geq 0 ; \\
P_{n, 1}(u)=\lim _{t \rightarrow \infty} P_{n, 1}(u, t-), \quad n \geq 0 ; \\
P_{n, 2}(u)=\lim _{t \rightarrow \infty} P_{n, 2}(u, t-), \quad n=a-1, c-1 .
\end{gathered}
$$

\section{Steady State Distributions}

To obtain the queue length distributions, we develop the difference equations using the remaining interarrival time as the supplementary variable. It may be noted that changes in the state of the system occur due to the following events: (i) an arrival, (ii) a service completion, (iii) a vacation completion, (iv) an arrival during changeover times, and (iv) completion of changeover times. Based on these events, observing the state of the system at two consecutive time epochs $t-$ and $(t+1)-$, using probabilistic arguments and the concept of stochastic balance at steady state, for $u \geq 1$ we have the following difference equations under the steady state condition $\rho<1$ :

$$
\begin{gathered}
P_{0,0}(u-1)=P_{0,0}(u)+\mu_{1} P_{0,1}(u), \\
P_{n, 0}(u-1)=P_{n, 0}(u)+\mu_{1} P_{n, 1}(u)+a_{u} P_{n-1,0}(0) \\
+\mu_{1} a_{u} P_{n-1,1}(0), \quad 1 \leq n \leq a-2, \\
P_{a-1,0}(u-1)=P_{a-1,0}(u)+\alpha_{1} P_{a-1,2}(u)+a_{u} P_{a-2,0}(0) \\
+\alpha_{1} \mu_{1} P_{a-1,1}(u)+\alpha_{1} \mu_{1} a_{u} P_{a-2,1}(0),
\end{gathered}
$$




$$
\begin{aligned}
P_{n, 0}(u-1)= & P_{n, 0}(u)+a_{u} P_{n-1,0}(0), \quad a \leq n \leq c-2 \\
P_{c-1,0}(u-1)= & \bar{\phi} P_{c-1,0}(u)+\bar{\phi} a_{u} P_{c-2,0}(0)+\bar{\phi} \alpha_{2} P_{c-1,2}(u) \\
+ & \alpha_{2} \phi P_{c-1,0}(u)+\alpha_{2} \phi a_{u} P_{c-2,0}(0) \\
P_{n, 0}(u-1)= & \bar{\phi} P_{n, 0}(u)+\bar{\phi} a_{u} P_{n-1,0}(0) \\
P_{0,1}(u-1)= & \bar{\mu}_{1} P_{0,1}(u)+a_{u} P_{a-1,2}(0)+a_{u} P_{c-1,2}(0) \\
& +\mu_{2} \sum_{n=a}^{b} P_{n, 1}(u)+\mu_{2} a_{u} \sum_{n=a}^{b} P_{n-1,1}(0) \\
& +\phi \sum_{n=c}^{b} P_{n, 0}(u)+\phi a_{u} \sum_{n=c}^{b} P_{n-1,0}(0) \\
& +\bar{\alpha}_{2} \phi a_{u} P_{c-2,0}(0)+\phi \alpha_{2} P_{c-1,2}(u) \\
P_{c-1,2}(u-1)= & \bar{\alpha}_{2} P_{c-1,2}(u)+\bar{\alpha}_{2} \phi P_{c-1,0}(u)+\bar{\mu}_{1} a_{u} P_{n-1,1}(0)+\mu_{2} P_{n+b, 1}(u) \\
& +\phi a_{u} P_{n+b-1,0}(0), \quad n \geq a \\
& +\phi P_{n+b, 0}(u)+\mu_{2} a_{u} P_{n+b-1,1}(0) \\
& +\phi a_{u} P_{n+b-1,0}(0) \\
& \bar{\mu}_{2} P_{n, 1}(u)+\bar{\mu}_{2} a_{u} P_{n-1,1}(0)+\mu_{2} P_{n+b, 1}(u) \\
+ & \phi P_{n+b, 0}(u)+\mu_{2} a_{u} P_{n+b-1,1}(0) \\
P_{n, 1}(u-1) & \bar{\alpha}_{1} P_{a-1,2}(u)+\bar{\alpha}_{1} \mu_{1} P_{a-1,1}(u)
\end{aligned}
$$

Let us define $P_{n, j}^{*}(z)=\sum_{u=0}^{\infty} P_{n, j}(u) z^{u}, n \geq 0$ and $j=0,1,2$, so that $P_{n, j}^{*}(1) \equiv P_{n, j}$ are the steady state probabilities that $n$ customers present in the queue and the server is in state $j$ at an arbitrary epoch. Multiplying (4) to (14) by $z^{u}$ and summing over $u$ from 1 to $\infty$, we obtain

$$
\begin{aligned}
(z-1) P_{0,0}^{*}(z)= & \mu_{1} P_{0,1}^{*}(z)-\mu_{1} P_{0,1}(0)-P_{0,0}(0) \\
(z-1) P_{n, 0}^{*}(z) & \\
= & \mu_{1} P_{n, 1}^{*}(z)+A(z)\left(P_{n-1,0}(0)+\mu_{1} P_{n-1,1}(0)\right) \\
& -\mu_{1} P_{n, 1}(0)-P_{n, 0}(0), \quad 1 \leq n \leq a-2 \\
(z-1) P_{a-1,0}^{*}(z)= & \alpha_{1} P_{a-1,2}^{*}(z)+A(z) P_{a-2,0}(0) \\
& +\alpha_{1} \mu_{1} P_{a-1,1}^{*}(z)+\alpha_{1} \mu_{1} A(z) P_{a-2,1}(0) \\
& -\alpha_{1} P_{a-1,2}(0)-\alpha_{1} \mu_{1} P_{a-1,1}(0) \\
& -P_{a-1,0}(0),
\end{aligned}
$$

$$
\begin{array}{r}
(z-1) P_{n, 0}^{*}(z)=A(z) P_{n-1,0}(0)-P_{n, 0}(0), \\
a \leq n \leq c-2,
\end{array}
$$

$$
\begin{aligned}
(z-\bar{\phi}) P_{c-1,0}^{*}(z)= & \bar{\phi} A(z) P_{c-2,0}(0)+\bar{\phi} \alpha_{2} P_{c-1,2}^{*}(z) \\
& +\alpha_{2} \phi P_{c-1,0}^{*}(z)+\alpha_{2} \phi A(z) P_{c-2,0}(0) \\
& -\bar{\phi} \alpha_{2} P_{c-1,2}(0)-\alpha_{2} \phi P_{c-1,0}(0) \\
& -\bar{\phi} P_{c-1,0}(0)
\end{aligned}
$$

$$
(z-\bar{\phi}) P_{n, 0}^{*}(z)=\bar{\phi} A(z) P_{n-1,0}(0)-\bar{\phi} P_{n, 0}(0), \quad n \geq c,
$$

$$
\begin{aligned}
\left(z-\overline{\mu_{1}}\right) & P_{0,1}^{*}(z) \\
= & A(z) P_{a-1,2}(0)+A(z) P_{c-1,2}(0) \\
& +\mu_{2} \sum_{n=a}^{b}\left(P_{n, 1}^{*}(z)+A(z) P_{n-1,1}(0)-P_{n, 1}(0)\right) \\
& -\bar{\mu}_{1} P_{0,1}(0) \\
& +\phi \sum_{n=c}^{b}\left(P_{n, 0}^{*}(z)+A(z) P_{n-1,0}(0)-P_{n, 0}(0)\right),
\end{aligned}
$$

$$
\begin{aligned}
(z- & \left.\mu_{1}\right) P_{n, 1}^{*}(z) \\
= & \bar{\mu}_{1} A(z) P_{n-1,1}(0)+\mu_{2} P_{n+b, 1}^{*}(z) \\
& +\phi P_{n+b, 0}^{*}(z)+\mu_{2} A(z) P_{n+b-1,1}(0)-\mu_{2} P_{n+b, 1}(0) \\
& +\phi A(z) P_{n+b-1,0}(0)-\phi P_{n+b, 0}(0)-\bar{\mu}_{1} P_{n, 1}(0)
\end{aligned}
$$$$
1 \leq n \leq a-1,
$$

$$
\begin{aligned}
& \left(z-\overline{\mu_{2}}\right) P_{n, 1}^{*}(z) \\
& =\bar{\mu}_{2} A(z) P_{n-1,1}(0)+\mu_{2} P_{n+b, 1}^{*}(z) \\
& \quad+\phi P_{n+b, 0}^{*}(z)+\mu_{2} A(z) P_{n+b-1,1}(0) \\
& +\phi A(z) P_{n+b-1,0}(0)-\mu_{2} P_{n+b, 1}(0)-\phi P_{n+b, 0}(0) \\
& -\bar{\mu}_{2} P_{n, 1}(0), \quad n \geq a, \\
& \left(z-\bar{\alpha}_{1}\right) P_{a-1,2}^{*}(z) \\
& =\bar{\alpha}_{1} \mu_{1} P_{a-1,1}^{*}(z)+\bar{\alpha}_{1} \mu_{1} A(z) P_{a-2,1}(0) \\
& \quad-\bar{\alpha}_{1} \mu_{1} P_{a-1,1}(0)-\bar{\alpha}_{1} P_{a-1,2}(0), \\
& \left(z-\bar{\alpha}_{2}\right) P_{c-1,2}^{*}(z) \\
& =\bar{\alpha}_{2} \phi P_{c-1,0}^{*}(z)+\bar{\alpha}_{2} \phi A(z) P_{c-2,0}(0)
\end{aligned}
$$




$$
\begin{aligned}
& +\phi \alpha_{2} P_{c-1,2}^{*}(z) \\
& -\bar{\alpha}_{2} \phi P_{c-1,0}(0)-\phi \alpha_{2} P_{c-1,2}(0)-\bar{\alpha}_{2} P_{c-1,2}(0)
\end{aligned}
$$

One important result is stated below in the form of a lemma using (15) to (25).

Lemma 1. The mean number of entrances into the system per unit time equals the mean arrival rate that is

$$
\sum_{n=0}^{\infty}\left(P_{n, 0}(0)+P_{n, 1}(0)\right)+P_{a-1,2}(0)+P_{c-1,2}(0)=\lambda .
$$

Proof. Adding (15) to (25), we get

$$
\begin{gathered}
\sum_{n=0}^{\infty}\left(P_{n, 0}^{*}(z)+P_{n, 1}^{*}(z)\right)+P_{a-1,2}^{*}(z)+P_{c-1,2}^{*}(z) \\
=\frac{A(z)-1}{z-1}\left\{\sum_{n=0}^{\infty}\left(P_{n, 0}(0)+P_{n, 1}(0)\right)\right. \\
\left.+P_{a-1,2}(0)+P_{c-1,2}(0)\right\} .
\end{gathered}
$$

Taking limit as $z \rightarrow 1$ and using the normalization condition, we get the result of the lemma.

3.1. Steady State Distribution at Prearrival Epochs. Let $P_{n, 0}^{-}$be the probability that $n(n \geq 0)$ customers are in the queue at prearrival epoch and the server is on vacation, $P_{n, 1}^{-}$denotes the probability of $n(n \geq 0)$ customers in the queue at prearrival epoch and the server is in busy period, and $P_{a-1,2}^{-}$ $\left(P_{c-1,2}^{-}\right)$denotes the probability of $a-1(c-1)$ customers in the queue at prearrival epoch and the server is in changeover time. Using Baye's theorem and (26), we express the prearrival epoch probabilities as below:

$$
\begin{aligned}
& P_{n, 0}^{-}=\frac{1}{\lambda} P_{n, 0}(0), \quad n \geq 0 ; \quad P_{n, 1}^{-}=\frac{1}{\lambda} P_{n, 1}(0), \quad n \geq 0 ; \\
& P_{a-1,2}^{-}=\frac{1}{\lambda} P_{a-1,2}(0) ; \quad P_{c-1,2}^{-}=\frac{1}{\lambda} P_{c-1,2}(0) .
\end{aligned}
$$

To obtain $P_{n, 0}^{-}, P_{n, 1}^{-}, P_{a-1,2}^{-}$, and $P_{c-1,2}^{-}$we need to evaluate $P_{n, 0}(0), P_{n, 1}(0), P_{a-1,2}(0)$, and $P_{c-1,2}(0)$ which is discussed below.

From (20), setting $z=\bar{\phi}$ and taking $k=P_{c-1,0}(0), r=$ $A(\bar{\phi})$ we get

$$
P_{n, 0}(0)=k r^{n-c+1}, \quad n \geq c-1 .
$$

Again from (20), we have

$$
P_{n, 0}^{*}(z)=\frac{\bar{\phi} k r^{n-c}(A(z)-r)}{z-\bar{\phi}}, \quad n \geq c .
$$

From (18), setting $z=1$ we have

$$
P_{a-1,0}(0)=P_{a, 0}(0)=\cdots=P_{c-2,0}(0) .
$$

We define the displacement operator $E$ as $E^{x} \omega_{n}=\omega_{n+x}$ (see $[19$, pp. 172]) and rewrite (23) as

$$
\begin{aligned}
\left(z-\bar{\mu}_{2}-\mu_{2} E^{b}\right) P_{n, 1}^{*}(z) & \left(\bar{\mu}_{2}+\mu_{2} E^{b}\right)\left(A^{*}(z)-E\right) P_{n-1,1}(0) \\
& +\frac{\phi k r^{n+b-c}(A(z)-r) z}{z-\bar{\phi}}, \quad n \geq a .
\end{aligned}
$$

Setting $z=\bar{\mu}_{2}+\mu_{2} E^{b}$, we get

$$
P_{n, 1}(0)=k_{1} \xi^{n}-\frac{\phi k r^{n+b-c+1}}{\bar{\mu}_{2}+\mu_{2} r^{b}-\bar{\phi}}, \quad n \geq a-1,
$$

where $k_{1}$ is an arbitrary constant and $\xi$ is a real root inside the unit circle of the equation $A\left(\bar{\mu}_{2}+\mu_{2} y^{b}\right)-y=0$ (from Rouche's theorem).

From (32) and (33), it follows that

$$
\begin{aligned}
P_{n, 1}^{*}(z)= & \frac{\left(\bar{\mu}_{2}+\mu_{2} \xi^{b}\right)(A(z)-\xi) k_{1} \xi^{n-1}}{z-\bar{\mu}_{2}-\mu_{2} \xi^{b}} \\
& -\frac{\bar{\phi} \phi k r^{n+b-c}(A(z)-r)}{(z-\bar{\phi})\left(\bar{\mu}_{2}+\mu_{2} r^{b}-\bar{\phi}\right)}, \quad n \geq a .
\end{aligned}
$$

Setting $z=\bar{\mu}_{1}$ in (22), we have

$$
P_{n, 1}(0)=k_{1} \tau_{1}(n)+k \tau_{2}(n), \quad 0 \leq n \leq a-2,
$$

where

$$
\begin{gathered}
\tau_{1}(n)=\frac{\xi^{a-1}\left(\bar{\mu}_{1}-\bar{\mu}_{2}-\mu_{2} A\left(\bar{\mu}_{1}\right)^{a-1-n} \xi^{b-a+1+n}\right)}{A\left(\bar{\mu}_{1}\right)^{a-1-n}\left(\bar{\mu}_{1}-\bar{\mu}_{2}-\mu_{2} \xi^{b}\right)}, \\
\tau_{2}(n) \\
=\frac{\phi r^{b-c+1+n}\left(r^{a-1-n}\left(\bar{\mu}_{2}-\bar{\mu}_{1}\right)+A\left(\bar{\mu}_{1}\right)^{a-1-n}\left(\bar{\phi}-\bar{\mu}_{2}\right)\right)}{A\left(\bar{\mu}_{1}\right)^{a-1-n}\left(\bar{\mu}_{1}-\bar{\phi}\right)\left(\bar{\mu}_{2}+\mu_{2} r^{b}-\bar{\phi}\right)} .
\end{gathered}
$$

Using (29), (30), (33), (34), and (35) in (22), we get

$$
P_{n, 1}^{*}(z)=k_{1} f_{1}(n, z)+k f_{2}(n, z), \quad 1 \leq n \leq a-1,
$$

where

$$
\begin{gathered}
f_{1}(n, z)=\frac{1}{z-\bar{\mu}_{1}}\left(\bar{\mu}_{1} A(z) \tau_{1}(n-1)-\bar{\mu}_{1} \tau_{1}(n)\right. \\
\left.+\frac{\xi^{n+b-1} \mu_{2} z(A(z)-\xi)}{z-\bar{\mu}_{2}-\mu_{2} \xi^{b}}\right), \\
f_{2}(n, z)=\frac{1}{z-\bar{\mu}_{1}}\left(\bar{\mu}_{1} A(z) \tau_{2}(n-1)-\bar{\mu}_{1} \tau_{2}(n)\right. \\
\left.+\frac{\phi r^{n+b-c}\left(\bar{\mu}_{2}-\bar{\phi}\right) z(A(z)-r)}{(z-\bar{\phi})\left(\bar{\mu}_{2}+\mu_{2} r^{b}-\bar{\phi}\right)}\right) .
\end{gathered}
$$


Setting $z=\bar{\alpha}_{1}$ in (24) and using (33), (35), and (37), one can obtain $P_{a-1,2}(0)$ as

$$
P_{a-1,2}(0)=k_{1} L_{1}+k L_{2},
$$

where

$$
\begin{aligned}
& \quad L_{1}=\mu_{1}\left(f_{1}\left(a-1, \bar{\alpha}_{1}\right)+A\left(\bar{\alpha}_{1}\right) \tau_{1}(a-2)-\xi^{a-1}\right), \\
& L_{2} \\
& =\mu_{1}\left(f_{2}\left(a-1, \bar{\alpha}_{1}\right)+A\left(\bar{\alpha}_{1}\right) \tau_{2}(a-2)+\frac{\phi r^{a+b-c}}{\bar{\mu}_{2}+\mu_{2} r^{b}-\bar{\phi}}\right) .
\end{aligned}
$$

Now inserting $z=\bar{\mu}_{1}$ in (21), we get

$$
P_{c-1,2}(0)=k_{1} L_{3}+k L_{4} \text {, }
$$

where

$$
\begin{aligned}
& L_{3}=\frac{1}{A\left(\bar{\mu}_{1}\right)}\left(\bar{\mu}_{1} \tau_{1}(0)-A\left(\bar{\mu}_{1}\right) L_{1}\right. \\
& \left.\quad-\frac{\mu_{2} \bar{\mu}_{1}\left(\xi^{a-1}-\xi^{b}\right)\left(A\left(\bar{\mu}_{1}\right)-\xi\right)}{\left(\bar{\mu}_{1}-\bar{\mu}_{2}-\mu_{2} \xi^{b}\right)(1-\xi)}\right), \\
& L_{4}=\frac{1}{A\left(\bar{\mu}_{1}\right)} \\
& \quad \times\left(\bar{\mu}_{1} \tau_{2}(0)\right. \\
& \quad+\frac{\mu_{2} \phi \bar{\mu}_{1}\left(r^{a+b-c}-r^{2 b-c+1}\right)\left(A\left(\bar{\mu}_{1}\right)-r\right)}{(1-r)\left(\bar{\mu}_{2}+\mu_{2} r^{b}-\bar{\phi}\right)\left(\bar{\mu}_{1}-\bar{\phi}\right)} \\
& \left.\quad-\frac{\bar{\mu}_{1} \phi\left(A\left(\bar{\mu}_{1}\right)-r\right)\left(1-r^{b-c+1}\right)}{\left(\bar{\mu}_{1}-\bar{\phi}\right)(1-r)}-A\left(\bar{\mu}_{1}\right) L_{2}\right) .
\end{aligned}
$$

Equation (24) together with (33), (35), (37), and (39) yields

$$
P_{a-1,2}^{*}(z)=k_{1} h_{1}(z)+k h_{2}(z),
$$

where

$$
\begin{gathered}
h_{1}(z)=\frac{\bar{\alpha}_{1}}{z-\bar{\alpha}_{1}}\left(\mu_{1} f_{1}(a-1, z)+\mu_{1} A(z) \tau_{1}(a-2)\right. \\
\left.-\mu_{1} \xi^{a-1}-L_{1}\right), \\
h_{2}(z)=\frac{\bar{\alpha}_{1}}{z-\bar{\alpha}_{1}}\left(\mu_{1} f_{2}(a-1, z)+\mu_{1} A(z) \tau_{2}(a-2)\right. \\
\left.+\frac{\mu_{1} \phi r^{a+b-c}}{\bar{\mu}_{2}+\mu_{2} r^{b}-\bar{\phi}}-L_{2}\right) .
\end{gathered}
$$

Setting $z=1$ in (21), we have

$$
P_{0,1}=\frac{1}{\mu_{1}}\left(L_{5} k_{1}+L_{6} k\right),
$$

where

$$
\begin{aligned}
L_{5}= & L_{1}+L_{3}+\frac{\xi^{a-1}-\xi^{b}}{1-\xi^{b}}-\bar{\mu}_{1} \tau_{1}(0), \\
L_{6}= & L_{2}+L_{4}-\frac{\mu_{2}\left(r^{a+b-c}-r^{2 b-c+1}\right)}{\bar{\mu}_{2}+\mu_{2} r^{b}-\bar{\phi}}+1 \\
& -r^{b-c+1}-\bar{\mu}_{1} \tau_{2}(0) .
\end{aligned}
$$

Setting $z=1$ in (15), we get

$$
P_{0,0}(0)=k_{1}\left(L_{5}-\mu_{1} \tau_{1}(0)\right)+k\left(L_{6}-\mu_{1} \tau_{2}(0)\right) .
$$

Setting $z=1$ in (16), we obtain

$$
P_{n, 0}(0)=k_{1} \tau_{3}(n)+k \tau_{4}(n), \quad 1 \leq n \leq a-2,
$$

where

$$
\begin{gathered}
\tau_{3}(n)=L_{5}+\bar{\mu}_{1} \tau_{1}(0)-\tau_{1}(n)+\frac{\left(1-\xi^{n}\right) \xi^{b}}{1-\xi^{b}} \\
\tau_{4}(n)=L_{6}+\bar{\mu}_{1} \tau_{2}(0)-\tau_{2}(n) \\
+\frac{r^{b-c+1}\left(1-r^{n}\right)\left(\bar{\mu}_{2}-\bar{\phi}\right)}{\bar{\mu}_{2}+\mu_{2} r^{b}-\bar{\phi}} .
\end{gathered}
$$

Inserting $z=1$ in (17) and using (39), (43), and (48), we get

$$
P_{a-1,0}(0)=k_{1} L_{7}+k L_{8},
$$

where

$$
\begin{aligned}
& L_{7}=\alpha_{1}( h_{1}(1)+\mu_{1} f_{1}(a-1,1) \\
&\left.+\mu_{1} \tau_{1}(a-2)-L_{1}-\mu_{1} \xi^{a-1}\right) \\
&+\tau_{3}(a-2), \\
& L_{8}=\alpha_{1}\left(h_{2}(1)+\mu_{1} f_{2}(a-1,1)+\mu_{1} \tau_{2}(a-2)-L_{2}\right.
\end{aligned}
$$

$$
\left.+\frac{\mu_{1} \phi r^{a+b-c}}{\bar{\mu}_{2}+\mu_{2} r^{b}-\bar{\phi}}\right)+\tau_{4}(a-2) .
$$

From this equation and (31) it follows that

$$
P_{n, 0}(0)=k_{1} L_{7}+k L_{8}, \quad a-1 \leq n \leq c-2 .
$$

Using the normalization condition, we have

$$
k_{1} L_{9}+k L_{10}=\lambda \text {, }
$$

where

$$
\begin{aligned}
L_{9}= & L_{1}+L_{3}+(c-a) L_{7}+\frac{\xi^{a-1}}{1-\xi}+\sum_{n=0}^{a-2}\left(\tau_{1}(n)+\tau_{3}(n)\right), \\
L_{10}= & L_{2}+L_{4}+(c-a) L_{8}+\frac{1}{1-r} \\
& -\frac{\phi r^{a+b-c}}{\left(\bar{\mu}_{2}+\mu_{2} r^{b}-\bar{\phi}\right)(1-r)}+\sum_{n=0}^{a-2}\left(\tau_{2}(n)+\tau_{4}(n)\right) .
\end{aligned}
$$


Setting $z=\bar{\alpha}_{2} \bar{\phi}+\alpha_{2} \phi$ in (19) and (25) and after simplification, we get

$$
k_{1} L_{11}+k L_{12}=0
$$

where

$$
\begin{aligned}
& L_{11}=\bar{\alpha}_{2} \phi A\left(\bar{\alpha}_{2} \bar{\phi}+\alpha_{2} \phi\right) L_{7}+\bar{\phi} \alpha_{2} L_{3}, \\
& L_{12}=\bar{\alpha}_{2} \phi A\left(\bar{\alpha}_{2} \bar{\phi}+\alpha_{2} \phi\right) L_{8}+\bar{\phi} \alpha_{2} L_{4}-\bar{\alpha}_{2} \phi .
\end{aligned}
$$

Combining (53) and (55), we can obtain the unknown constants $k_{1}$ and $k$ as

$$
k_{1}=\frac{-\lambda L_{12}}{L_{11} L_{10}-L_{9} L_{12}}, \quad k=\frac{\lambda L_{11}}{L_{11} L_{10}-L_{9} L_{12}} .
$$

Theorem 2. The prearrival epoch queue length distributions $P_{n, j}^{-}$that an arrival sees $n$ customers in the queue and the server is in state $j(=0,1,2)$ are given by

$$
\begin{aligned}
P_{n, 0}^{-} & =\frac{1}{\lambda}\left[k_{1} \tau_{3}(n)+k \tau_{4}(n)\right], \quad 0 \leq n \leq a-2, \\
P_{n, 0}^{-} & =\frac{1}{\lambda}\left[k_{1} L_{7}+k L_{8}\right], \quad a-1 \leq n \leq c-2, \\
P_{n, 0}^{-} & =\frac{1}{\lambda} k r^{n-c+1}, \quad n \geq c-1, \\
P_{n, 1}^{-} & =\frac{1}{\lambda}\left[k_{1} \tau_{1}(n)+k \tau_{2}(n)\right], \quad 0 \leq n \leq a-2, \\
P_{n, 1}^{-} & =\frac{1}{\lambda}\left[k_{1} \xi^{n}-\frac{\phi k r^{n+b-c+1}}{\bar{\mu}_{2}+\mu_{2} r^{b}-\bar{\phi}}\right], \quad n \geq a-1, \\
P_{a-1,2}^{-} & =\frac{1}{\lambda}\left[k_{1} L_{1}+k L_{2}\right], \\
P_{c-1,2}^{-} & =\frac{1}{\lambda}\left[k_{1} L_{3}+k L_{4}\right] .
\end{aligned}
$$

Proof. Using (28) in (47)-(52), (29), (35), (33), (39), and (41), we obtain the result of the theorem.

3.2. Steady State Distribution at Arbitrary Epochs. To obtain the queue length distributions at arbitrary epochs $P_{n, j}(n \geq$ $0, j=0,1,2)$, we use $P_{n, j}^{*}(1)=P_{n, j}$ and the rate probabilities $P_{n, j}(0)$. This is discussed in the following theorems.
Theorem 3. The arbitrary epoch probabilities are given by

$$
\begin{gathered}
P_{n, 0}=\frac{\bar{\phi} k r^{n-c}(1-r)}{\phi}, \quad n \geq c, \\
P_{n, 1}=k_{1} f_{1}(n, 1)+k f_{2}(n, 1), \quad 1 \leq n \leq a-2, \\
P_{a-1,1}=k_{1} f_{1}(a-1,1)+k f_{2}(a-1,1), \\
P_{n, 1}=\frac{\left(\bar{\mu}_{2}+\mu_{2} \xi^{b}\right)(1-\xi) k_{1} \xi^{n-1}}{\mu_{2}\left(1-\xi^{b}\right)} \\
\quad-\frac{\bar{\phi}_{k} r^{n+b-c}(1-r)}{\bar{\mu}_{2}+\mu_{2} r^{b}-\bar{\phi}}, \quad n \geq a, \\
P_{a-1,2}=k_{1} h_{1}(1)+k h_{2}(1) .
\end{gathered}
$$

Proof. Setting $z=1$ in (30), (37), (34), and (43), we get (59)(63), respectively.

One may note here that from Theorem 3 we cannot get $\left\{P_{n, 0}\right\}_{0}^{c-1}, P_{0,1}$, and $P_{c-1,2}$. We have already obtained $P_{0,1}$ in (45) and the remaining can be obtained using the following theorem.

Theorem 4. The arbitrary epoch probabilities $\left\{P_{n, 0}\right\}_{0}^{c-1}$ and $P_{c-1,2}$ are given by

$$
\begin{gathered}
P_{0,0}=\mu_{1} P_{0,1}^{*(1)}(1)=k_{1} L_{13}+k L_{14}, \\
P_{n, 0}=k_{1}\left[\mu_{1} \frac{\partial f_{1}}{\partial z}(n, 1)+\frac{1}{\lambda}\left(\mu_{1} \tau_{1}(n-1)+\tau_{3}(n-1)\right)\right] \\
+k\left[\mu_{1} \frac{\partial f_{2}}{\partial z}(n, 1)+\frac{1}{\lambda}\left(\mu_{1} \tau_{2}(n-1)+\tau_{4}(n-1)\right)\right] \\
P_{a-1,0}=k_{1}\left[\alpha_{1} h_{1}^{(1)}(1)+\alpha_{1} \mu_{1} f_{1}^{(1)}(a-1,1)\right. \\
\left.+\frac{\alpha_{1} \mu_{1}}{\lambda} \tau_{1}(a-2)+\frac{1}{\lambda} \tau_{3}(a-2)\right] \\
+k\left[\alpha_{1} h_{2}^{(1)}(1)+\alpha_{1} \mu_{1} f_{2}^{(1)}(a-1,1)\right. \\
\left.+\frac{\alpha_{1} \mu_{1}}{\lambda} \tau_{2}(a-2)+\frac{1}{\lambda} \tau_{4}(a-2)\right] \\
P_{n, 0}=\frac{1}{\lambda}\left(k_{1} L_{7}+k L_{8}\right), \quad a \leq n \leq c-2
\end{gathered}
$$




$$
\begin{aligned}
& P_{c-1,0} \\
& =\frac{1}{\bar{\alpha}_{2} \phi+\alpha_{2} \bar{\phi}} \\
& \times\left[k_{1}\left(\frac{\alpha_{2} \bar{\phi}-\bar{\alpha}_{2} \phi \lambda}{\lambda} L_{7}+\left(\phi \alpha_{2}+\bar{\alpha}_{2}\right) L_{3}\right)\right. \\
& \left.+k\left(\frac{\alpha_{2} \bar{\phi}-\bar{\alpha}_{2} \phi \lambda}{\lambda} L_{8}+\left(\phi \alpha_{2}+\bar{\alpha}_{2}\right) L_{4}+\bar{\alpha}_{2} \phi\right)\right], \\
& P_{c-1,2} \\
& =\frac{1}{\bar{\alpha}_{2} \phi+\alpha_{2} \bar{\phi}} \\
& \times\left[k_{1}\left(\frac{\bar{\alpha}_{2} \phi(1+\lambda)}{\lambda} L_{7}-\left(\phi \alpha_{2}+\bar{\alpha}_{2}\right) L_{3}\right)\right. \\
& \left.+k\left(\frac{\bar{\alpha}_{2} \phi(1+\lambda)}{\lambda} L_{8}-\left(\phi \alpha_{2}+\bar{\alpha}_{2}\right) L_{4}-\bar{\alpha}_{2} \phi\right)\right],
\end{aligned}
$$

where

$$
\begin{aligned}
L_{13}= & \frac{L_{1}}{\lambda}+\frac{L_{3}}{\lambda}-\frac{L_{5}}{\mu_{1}} \\
& +\frac{\left(\mu_{2}\left(1-\xi^{b}\right)-\lambda(1-\xi)\left(\bar{\mu}_{2}+\mu_{2} \xi^{b}\right)\right)\left(\xi^{a-1}-\xi^{b}\right)}{\lambda \mu_{2}(1-\xi)\left(1-\xi^{b}\right)^{2}} \\
L_{14}= & \frac{L_{2}}{\lambda}+\frac{L_{4}}{\lambda}-\frac{L_{6}}{\mu_{1}}+\frac{\bar{\phi} \phi-\bar{\phi} \lambda(1-r)+\phi^{2}}{\phi \lambda(1-r)} \\
& \times\left(1-r^{b-c+1}-\frac{\mu_{2}\left(r^{b+a-c}-r^{2 b-c+1}\right)}{\bar{\mu}_{2}+\mu_{2} r^{b}-\bar{\phi}}\right)
\end{aligned}
$$

and $(\partial f / \partial z)$ represents the partial derivative of $f$ with respect to $z$.

Proof. Differentiating (30) and (34) and setting $z=1$ yields

$$
\begin{gathered}
P_{n, 0}^{*(1)}(1)=\frac{k \bar{\phi} r^{n-c}(\phi-\lambda(1-r))}{\lambda \phi^{2}}, \quad n \geq c, \\
P_{n, 1}^{*(1)}(1)=\frac{\left(\bar{\mu}_{2}+\mu_{2} \xi^{b}\right) k_{1} \xi^{n-1}\left(\mu_{2}\left(1-\xi^{b}\right)-\lambda(1-\xi)\right)}{\lambda \mu_{2}^{2}\left(1-\xi^{b}\right)^{2}} \\
-\frac{\bar{\phi} k r^{n+b-c}(\phi-\lambda(1-r))}{\lambda \phi\left(\bar{\mu}_{2}+\mu_{2} r^{b}-\bar{\phi}\right)}, \quad n \geq a .
\end{gathered}
$$

Differentiating (15) and (21) and (16) to (18), setting = 1, and using (71), we get (64) to (67), respectively. we get

Adding and differentiating (19) and (25) and setting $z=1$,

$$
P_{c-1,0}+P_{c-1,2}=\frac{1}{\lambda} P_{c-2,0}(0) .
$$

Setting $z=1$ in (25) yields

$$
\begin{aligned}
-\bar{\alpha}_{2} \phi P_{c-1,0}+\alpha_{2} \bar{\phi} P_{c-1,2} & =\bar{\alpha}_{2} \phi P_{c-2,0}(0) \\
& -\bar{\alpha}_{2} \phi P_{c-1,0}(0) \\
& -\left(\phi \alpha_{2}+\bar{\alpha}_{2}\right) P_{c-1,2}(0) .
\end{aligned}
$$

On combining (72) and (73) one can obtain (68) and (69).

This completes the evaluation of the arbitrary epoch probabilities.

The following results can be deduced from our model by taking specific values of the parameters , $c, b, \alpha_{1}, \alpha_{2}$, and $\phi$.

Remark 5. Let $a=c=b=1, \mu_{1}=\mu_{2}$, and $\alpha_{1}, \alpha_{2} \rightarrow 1$, that is, the batch size is one and no changeover time. The model reduces to $G I / \mathrm{Geo} / 1 / \infty$ queue with multiple vacations and our results match numerically with Tian and Zhang [10].

Remark 6. Taking $a=c, \mu_{1}=\mu_{2}$, and $\alpha_{1}, \alpha_{2} \rightarrow 1$, the present model reduces to $G I / \mathrm{Geo}^{(a, b)} / 1 / \infty / M V$ queue.

Remark 7. $a=c=b=1, \mu_{1}=\mu_{2}$, and $\phi, \alpha_{1}, \alpha_{2} \rightarrow 1$, that is, the batch size is one, no vacation, and no changeover time. In this case, the system reduces to $G I / \mathrm{Geo} / 1 / \infty$ queue and our results match with the results available in the literature.

\section{Performance Measures and Cost Model}

Among the different features of queueing system, performance measures are very important since they reflect the effectiveness of the queueing system. Once the state probabilities at various epochs are known, we can evaluate some performance measures. The average queue length $\left(L_{q}\right)$ and the average waiting time in the queue $\left(W_{q}\right)$ of a customer using Little's rule are, respectively, given by

$$
\begin{aligned}
L_{q} & =\sum_{n=0}^{\infty} n\left(P_{n, 0}+P_{n, 1}\right)+(a-1) P_{a-1,2}+(c-1) P_{c-1,2}, \\
W_{q} & =\frac{L_{q}}{\lambda} .
\end{aligned}
$$

The probability that the server is in vacation $\left(P_{v}\right)$, the probability that the server is busy $\left(P_{b}\right)$, and the probability that the server is in changeover times $\left(P_{c}\right)$ are, respectively, given by

$$
P_{v}=\sum_{n=0}^{\infty} P_{n, 0}^{-}, \quad P_{b}=\sum_{n=0}^{\infty} P_{n, 1}^{-}, \quad P_{c}=P_{a-1,2}^{-}+P_{c-1,2}^{-} .
$$

4.1. Cost Model. We develop the total expected cost function per unit time with an objective to determine the optimum values of $\mu_{2}$ and $\phi$ so that the expected cost function is minimized. Let us define 
$C_{1}=$ the unit time cost of every waiting customer in the queue;

$C_{2}=$ service cost per unit time when the server is busy with $0 \leq n \leq a-1$ customers in the queue;

$C_{3}=$ service cost per unit time when the server is busy with $n \geq a$ customers in the queue;

$C_{4}=$ fixed cost per unit time when the server is on vacation;

$C_{5}=$ fixed cost per unit time when the server is in changeover time (after service completion epoch);

$C_{6}=$ fixed cost per unit time when the server is in changeover time (after vacation completion epoch).

Let $F$ be the total expected cost per unit time. Using the definitions of each cost element and its corresponding system characteristics, we have

$$
F=C_{1} L_{q}+C_{2} \mu_{1}+C_{3} \mu_{2}+C_{4} \phi+C_{5} \alpha_{1}+C_{6} \alpha_{2} .
$$

We have considered the following optimization problems.

(i) Minimize $F(\phi)$ subject to the constraint $0.07<\phi<$ 0.24 .

(ii) Minimize $F\left(\mu_{2}\right)$ subject to the constraint $0.06<\mu_{2}<$ 1.

It may be noted that the choice of bounds for the variables $\phi$ and $\mu_{2}$ is arbitrary. However, the upper bound for $\phi$ is chosen to be small $(<0.24)$ in order to emphasize the effect of vacation rate on the total expected cost numerically, as the larger values of $\phi$ indicates smaller vacation durations. The numerical searching approach is implemented using genetic algorithm on the computer software Mathematica with $\mu_{2}$ and $\phi$ as the decision variables.

\section{Numerical Results and Discussion}

To demonstrate the applicability of the theoretical investigation made in the previous sections, we present some numerical results in the form of tables and graphs. We have considered the following cost parameters: $C_{1}=15, C_{2}=30$, $C_{3}=50, C_{4}=35, C_{5}=5, C_{6}=8$. In addition to these cost parameters we have taken the parameters (i) $\phi=0.3$, $\alpha_{1}=0.35, \alpha_{2}=0.25, \lambda=0.2, \mu_{1}=0.05, \mu_{2}=0.1$ for Tables 1 and 2 and Figures 1-7, (ii) $a=4, c=7, b=10$ for Tables 1 and 2 and Figures 2-5, and (iii) $a=3, c=14, b=15$ for Figures 6 and 7, unless they are considered as variables or their values are mentioned in the respective figures and tables. It may be noted from Theorems $2-4$ that the evaluation of the stationary probabilities has the computational speed which is of linear order.

Using genetic algorithm, the best values of $\mu_{2}$ and the corresponding minimum expected cost $F^{*}$ for different values of $\lambda$ are shown in Table 1 for geometric interarrival times. One can see that as the mean arrival rate increases both the optimal mean service rate and the minimum cost increase. Table 2 presents the best values of $\phi^{*}$ and $F^{*}$ reached by $\mathrm{GA}$ for different interarrival time distributions and various

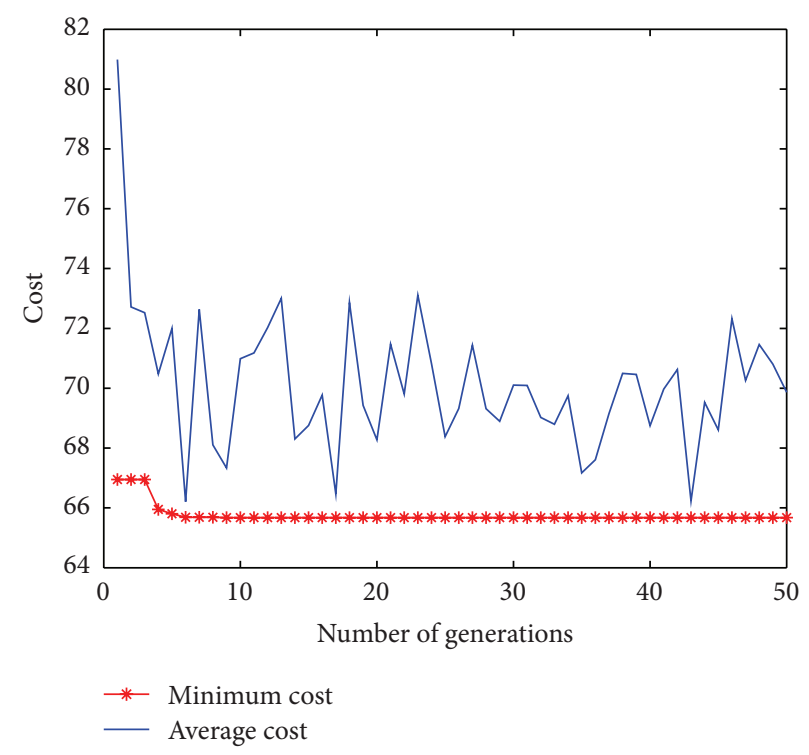

Figure 2: Cost versus generation.

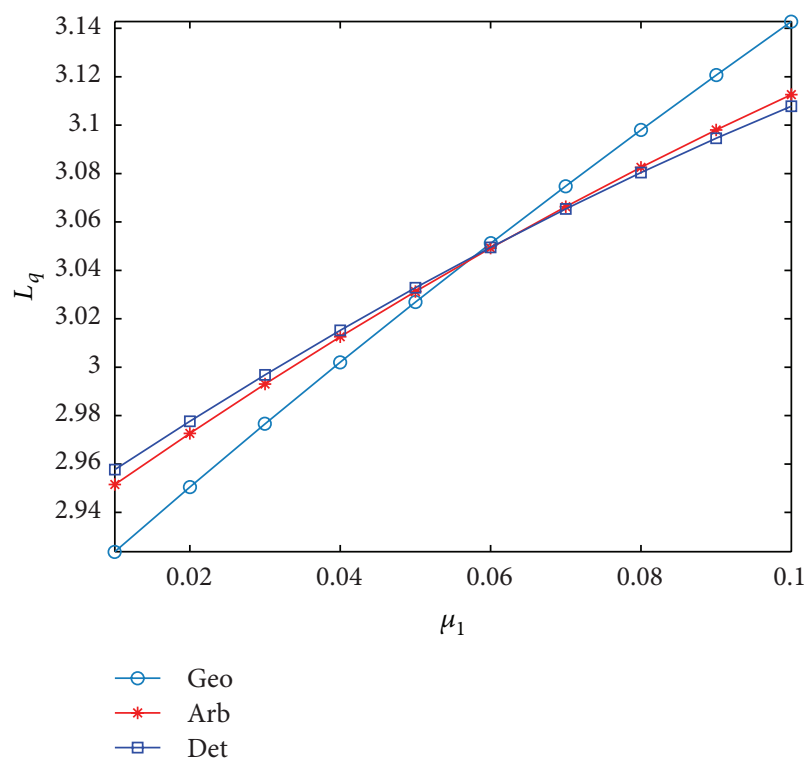

FigURE 3: Effect of $\mu_{1}$ on $L_{q}$.

values of $\rho$. The results shown in Table 2 indicate that there is a certain optimal value of $\rho$ (around 0.15 ) at which $F\left(\phi^{*}\right)$ is minimum.

Table 3 presents the sensitivity analysis of average queue length for the models with (i) multiple vacations and changeover times (MV, Chov), (ii) multiple vacations without changeover times (MV, No Chov), and (iii) no vacation and no changeover times (No vac, No Chov) with constant and varying service rate. We observe that $L_{q}$ increases with the increase of $a$ and $c$ and decreases as $b$ increases for all the cases. As the values of $a$ and $c$ increase, it results in greater accumulation of customers in the queue thereby increasing $L_{q}$. The expected queue length is larger for constant service 
TABLE 1: Best values of $\mu_{2}^{*}$ and $F^{*}$ reached by GA for different values of $\lambda$.

\begin{tabular}{lcccccc}
\hline$\lambda$ & 0.1 & 0.2 & 0.3 & 0.4 & 0.5 & 0.6 \\
\hline$\mu_{2}^{*}$ & 0.091711 & 0.125505 & 0.181864 & 0.242477 & 0.273388 & 0.31004 \\
$F^{*}$ & 64.2696 & 65.6766 & 67.7239 & 69.7738 & 71.6250 & 73.3880 \\
\hline
\end{tabular}

TABLE 2: Best values of $\phi^{*}$ and $F^{*}$ reached by GA for various values of $\rho$.

\begin{tabular}{|c|c|c|c|c|c|c|}
\hline \multirow{2}{*}{$\rho$} & \multicolumn{2}{|c|}{ Geometric } & \multicolumn{2}{|c|}{ Deterministic } & \multicolumn{2}{|c|}{ Arbitrary } \\
\hline & $\phi^{*}$ & $F^{*}$ & $\phi^{*}$ & $F^{*}$ & $\phi^{*}$ & $F^{*}$ \\
\hline 0.06 & 0.200074 & 71.6547 & 0.198892 & 72.5481 & 0.198577 & 72.4214 \\
\hline 0.08 & 0.198844 & 67.9247 & 0.196938 & 68.8081 & 0.197362 & 68.6826 \\
\hline 0.1 & 0.197485 & 65.9327 & 0.196496 & 66.7716 & 0.19625 & 66.6536 \\
\hline 0.15 & 0.193744 & 64.1857 & 0.192704 & 64.8441 & 0.193275 & 64.7552 \\
\hline 0.2 & 0.191291 & 64.5873 & 0.18969 & 65.0040 & 0.190088 & 64.9519 \\
\hline 0.25 & 0.188076 & 66.2148 & 0.186692 & 66.3301 & 0.186658 & 66.3228 \\
\hline 0.3 & 0.18469 & 68.8585 & 0.183194 & 68.6027 & 0.183564 & 68.6501 \\
\hline 0.35 & 0.181152 & 72.5531 & 0.179647 & 71.8410 & 0.179887 & 71.9552 \\
\hline 0.4 & 0.177438 & 77.4756 & 0.176406 & 76.2003 & 0.176153 & 76.3967 \\
\hline
\end{tabular}

TABLE 3: Sensitivity analysis of Geo/Geo ${ }^{(a, c, b)} / 1$ queue with and without state dependent, vacations, and changeover times.

\begin{tabular}{|c|c|c|c|c|c|c|c|}
\hline \multirow{5}{*}{$c$} & \multirow{5}{*}{$(a, b)$} & \multicolumn{6}{|c|}{$L_{q}$} \\
\hline & & \multicolumn{3}{|c|}{$\mu_{1}=0.05, \mu_{2}=0.1$} & \multicolumn{3}{|c|}{$\mu_{1}=\mu_{2}=0.05$} \\
\hline & & MV, Chov & MV, No Chov & No vac, & MV, Chov & MV, No Chov & No vac, \\
\hline & & $\phi=0.3, \alpha_{1}=0.35$ & $\phi=0.3$ & No Chov & $\phi=0.3, \alpha_{1}=0.35$ & $\phi=0.3$ & No Chov \\
\hline & & $\alpha_{2}=0.25, \lambda=0.2$ & $\lambda=0.2$ & $\lambda=0.2$ & $\alpha_{2}=0.25, \lambda=0.2$ & $\lambda=0.2$ & $\lambda=0.2$ \\
\hline \multirow{5}{*}{7} & $(3,10)$ & 2.81752 & 2.94114 & 2.76699 & 4.05474 & 4.11482 & 4.0067 \\
\hline & $(4,10)$ & 3.03040 & 3.14029 & 2.95542 & 4.09340 & 4.16072 & 4.03604 \\
\hline & $(5,10)$ & 3.20035 & 3.30077 & 3.10829 & 4.13791 & 4.20747 & 4.06983 \\
\hline & $(5,11)$ & 3.19120 & 3.29053 & 3.10034 & 4.04412 & 4.11406 & 3.97658 \\
\hline & $(5,12)$ & 3.18598 & 3.28500 & 3.09540 & 3.98130 & 4.05235 & 3.91305 \\
\hline \multirow{5}{*}{8} & $(3,10)$ & 3.13138 & 3.27399 & 3.08551 & 4.24377 & 4.32539 & 4.19104 \\
\hline & $(4,10)$ & 3.36391 & 3.49000 & 3.28921 & 4.31313 & 4.4003 & 4.24690 \\
\hline & $(5,10)$ & 3.54633 & 3.66195 & 3.45191 & 4.38036 & 4.46915 & 4.30085 \\
\hline & $(5,11)$ & 3.53526 & 3.64735 & 3.44485 & 4.29048 & 4.37541 & 4.21707 \\
\hline & $(5,12)$ & 3.52970 & 3.64070 & 3.44047 & 4.23238 & 4.31705 & 4.16008 \\
\hline \multirow{5}{*}{9} & $(3,10)$ & 3.47278 & 3.63592 & 3.42944 & 4.47623 & 4.58655 & 4.41201 \\
\hline & $(4,10)$ & 3.72724 & 3.87294 & 3.64835 & 4.58047 & 4.69580 & 4.49617 \\
\hline & $(5,10)$ & 3.92456 & 4.06050 & 3.82090 & 5.34441 & 4.79109 & 4.57118 \\
\hline & $(5,11)$ & 3.90616 & 4.03152 & 3.81461 & 4.57708 & 4.67891 & 4.49579 \\
\hline & $(5,12)$ & 3.89853 & 4.02055 & 3.81069 & 4.52012 & 4.61798 & 4.44461 \\
\hline
\end{tabular}

rate than that of state dependent service rate for each of the cases considered. This indicates that the model in which the service rate of the server depends on the queue size performs better. Further, for both constant and varying service rate, $L_{q}$ for case (iii) is smallest due to the absence of vacation but with the presence of vacation the one with changeover time (case (i)) showed better performance. This is because in case (ii) the server directly goes to vacation if the conditions on $a(c)$ are not met after service completion (vacation termination) epoch. Thus, the introduction of changeover policy forces the server to wait for some time in the system which results in decreasing the queue length.
Figure 2 depicts the minimum and average cost for number of generations considered in the GA, when $\mu_{2}$ is a decision variable and the interarrival time distribution is geometric. We observe that the method stabilizes for larger generation values. This is to say that even though the average cost varies widely from generation to generation the minimum cost becomes stable after some generations.

Figure 3 provides the expected number in the queue $L_{q}$ with the change of $\mu_{1}$ for different interarrival distributions. From the figure we observe that $L_{q}$ increases with $\mu_{1}$ for any interarrival time distribution. This shows that keeping the service rate lower whenever the queue size is less than 


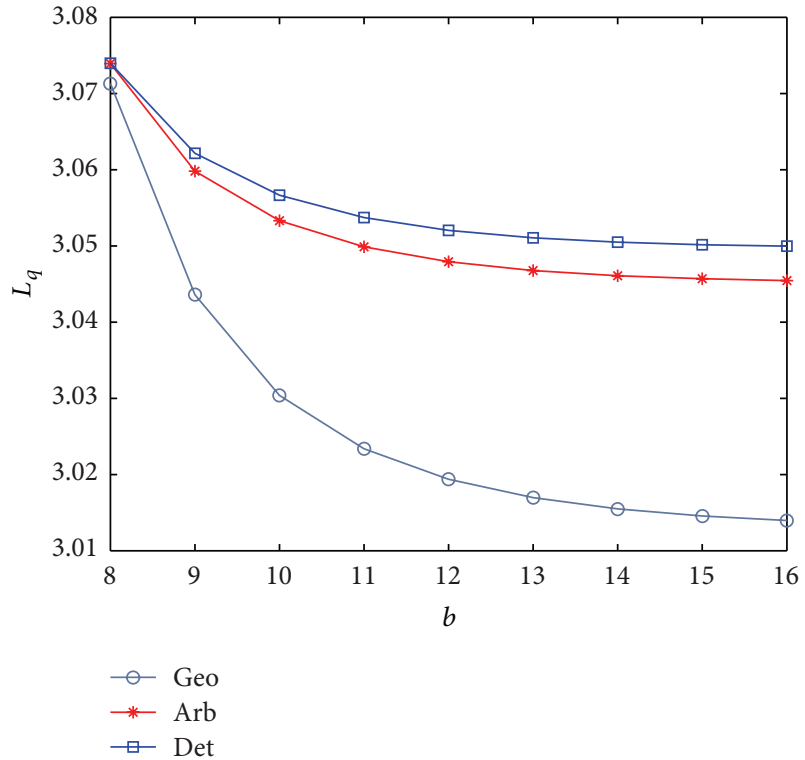

FIGURE 4: Effect of $b$ on $L_{q}$.

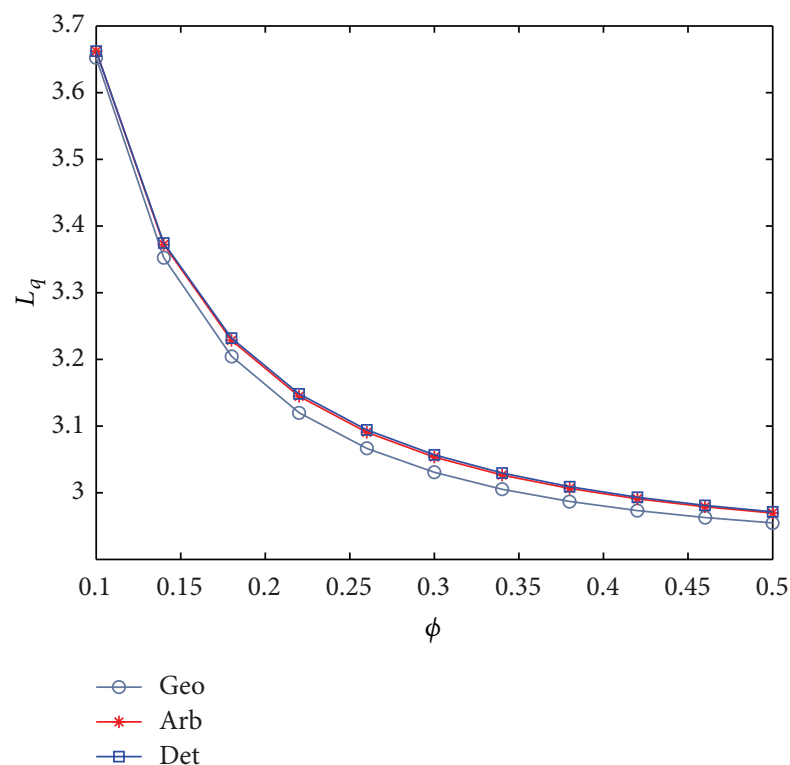

FIGURE 5: Effect of $\phi$ on $L_{q}$.

some specified value ( $a$ in this model) contributes for better performance.

Figure 4 shows the effect of $b$ on $L_{q}$ for different interarrival distributions. As expected, the average number of customers in the queue monotonically decreases as the maximum batch size increases. It can also be observed that in the case of geometric interarrival time distribution $L_{q}$ is lowest.

The effect of $\phi$ on $L_{q}$ for different interarrival time distributions is presented in Figure 5. We can see that $L_{q}$ decreases as $\phi$ increases. As $\phi$ increases the mean vacation time decreases which indicates that the server is available

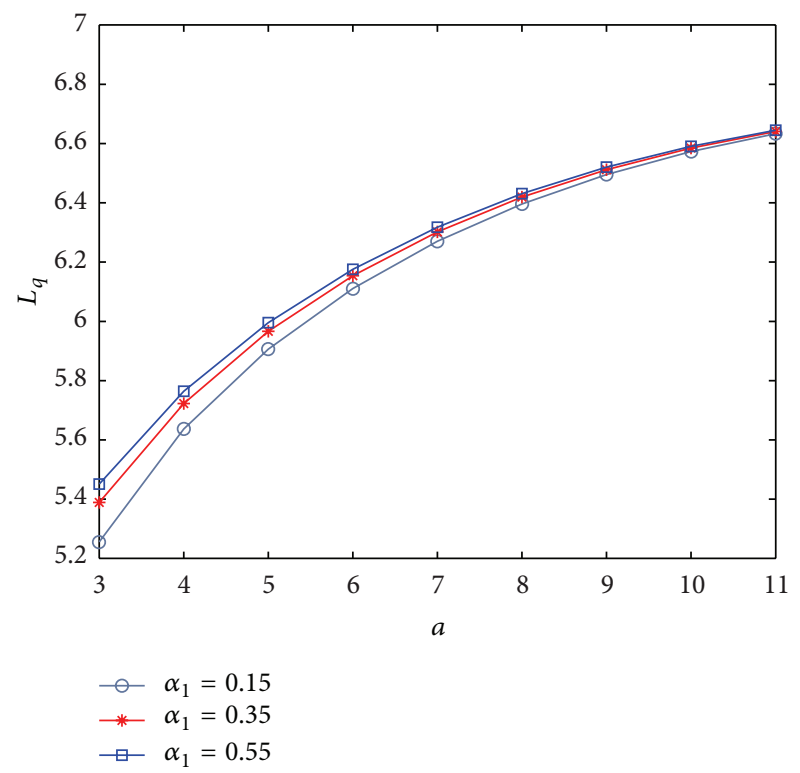

FigURE 6: Impact of $a$ on $L_{q}$.

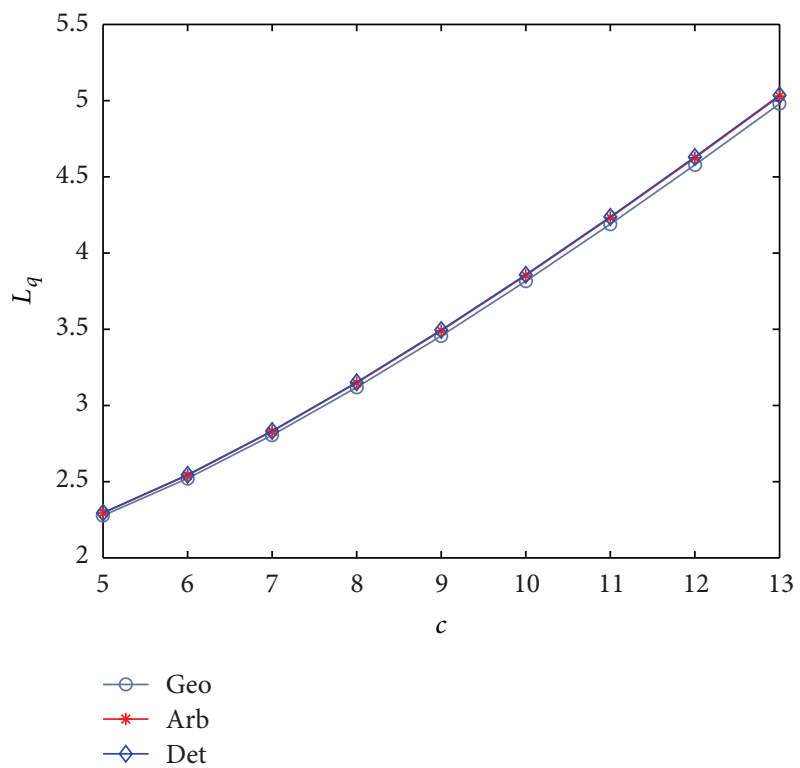

Figure 7: Effect of $c$ on $L_{q}$.

in the system more frequently, clearing the accumulated customers and as a result $L_{q}$ decreases. Figure 6 illustrates the effect of $a$ on $L_{q}$ for geometric interarrival time distribution for different values of $\alpha_{1}$. It is observed that $L_{q}$ increases as $a$ increases. Since $a$ is the minimum batch size, increasing $a$ will result in greater accumulation of customers in the queue which in turn results in increasing $L_{q}$. Further, we can see that $L_{q}$ increases as $\alpha_{1}$ increases for fixed $a$. This is due to the fact that as $\alpha_{1}$ gets larger the mean duration of changeover times becomes smaller so that the server will go for vacation without waiting for an arrival for some reasonable duration of time. This contributes for $L_{q}$ to increase. 
Figure 7 depicts the effect of $c$ on $L_{q}$ for different interarrival time distributions. It is observed that $L_{q}$ increases as $c$ increases. Since $c$ is the minimum threshold to start service, increasing $c$ will result in greater accumulation of customers in the queue thereby increasing $L_{q}$. Further, it is observed that there is no significant difference among the interarrival distributions considered.

\section{Conclusions}

In this paper, we have analyzed a discrete-time renewal input infinite buffer bulk service queue with multiple vacations and changeover times, where the service rate of the server depends on the queue size. We have obtained the queue length distributions at arbitrary and prearrival epochs. Utilizing these distributions, we have derived some important performance measures and a cost model is developed to determine the best values of service and vacation rates reached by genetic algorithm. Numerical illustrations in the form of tables and graphs are reported to demonstrate how various parameters of the model influence the behavior of the system. This study reveals that not only the interarrival times and vacation duration play an important role in queueing processes but also changeover times and state dependent services have a major role. The analytical approach used in this paper would be interesting to study discrete-time $G I / \mathrm{Geo}^{(a, c, b)} / 1$ queue with single (multiple) working vacation(s) and other vacation policies, which are left for future research work.

\section{Conflict of Interests}

The authors declare that there is no conflict of interests regarding the publication of this paper.

\section{References}

[1] H. Bruneel and B. G. Kim, Discrete-Time Models for Communication Systems Including ATM, Kluwer Academic Publishers, Boston, Mass, USA, 1993.

[2] H. Takagi, Queueing Analysis: A Foundation of Performance Evaluation. Vol. 3. Discrete-Time Systems, North-Holland, Amsterdam, The Netherlands, 1993.

[3] P. Tran-Gia, C. Blondia, and D. Towsley, "Discrete-time models and analysis methods," Performance Evaluation, vol. 21, no. 1-2, pp. 1-2, 1994.

[4] T. G. Robertazzi, Computer Networks and Systems: Queueing Theory and Performance Evaluation, Telecommunication Networks and Computer Systems, Springer, New York, NY, USA, 1990.

[5] J. Medhi, Stochastic Models in Queueing Theory, Academic Press, Boston, Mass, USA, 1991.

[6] M. L. Chaudhry and J. G. C. Templeton, A First Course in Bulk Queues, John Wiley \& Sons, New York, NY, USA, 1983.

[7] U. C. Gupta and V. Goswami, "Performance analysis of finite buffer discrete-time queue with bulk service," Computers \& Operations Research, vol. 29, no. 10, pp. 1331-1341, 2002.
[8] M. L. Chaudhry and S. H. Chang, "Analysis ofthe discretetime bulk-service queue $\mathrm{Geo} / \mathrm{G}^{\mathrm{Y}} / 1 / N+B$," Operations Research Letters, vol. 32, no. 4, pp. 355-363, 2004.

[9] V. Goswami, J. R. Mohanty, and S. K. Samanta, "Discretetime bulk-service queues with accessible and non-accessible batches," Applied Mathematics and Computation, vol. 182, no. 1, pp. 898-906, 2006.

[10] N. Tian and Z. G. Zhang, "The discrete-time GI/Geo/1 queue with multiple vacations," Queueing Systems, vol. 40, no. 3, pp. 283-294, 2002.

[11] V. Goswami and P. Vijaya Laxmi, "Discrete-time renewal input multiple vacation queue with accessible and non-accessible batches," Opsearch, vol. 48, no. 4, pp. 335-354, 2011.

[12] L. Tadj and C. Abid, "Optimal management policy for a single and bulk service queue under Bernoulli vacation schedules," International Journal of Applied Decision Sciences, vol. 2, no. 3, pp. 262-274, 2009.

[13] C. Baburaj, "A discrete time $(a, c, d)$ policy bulk service queue," International Journal of Information and Management Sciences, vol. 21, no. 4, pp. 469-480, 2010.

[14] X. Chao and A. Rahman, "Analysis and computational algorithm for queues with state-dependent vacations. I. G/M(n)/1/K," Journal of Systems Science \& Complexity, vol. 19, no. 1, pp. 36-53, 2006.

[15] P. R. Parthasarathy and R. Sudhesh, "Time-dependent analysis of a single-server retrial queue with state-dependent rates," Operations Research Letters, vol. 35, no. 5, pp. 601-611, 2007.

[16] J. H. Holland, Adaptation in Natural and Artificial Systems, University of Michigan Press, Ann Arbor, Mich, USA, 1975.

[17] R. L. Haupt and S. E. Haupt, Practical Genetic Algorithms, John Wiley \& Sons, Hoboken, NJ, USA, 2nd edition, 2004.

[18] S. S. Rao, Engineering Optimization: Theory and Practice, John Wiley \& Sons, New York, NY, USA, 20009.

[19] N. Tian and Z. G. Zhang, Vacation Queueing Models: Theory and Applications, International Series in Operations Research \& Management Science, 93, Springer, New York, NY, USA, 2006. 


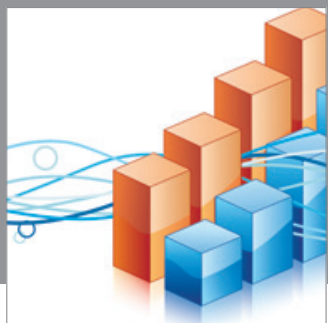

Advances in

Operations Research

mansans

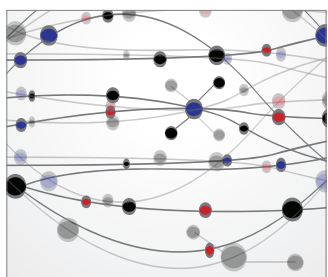

The Scientific World Journal
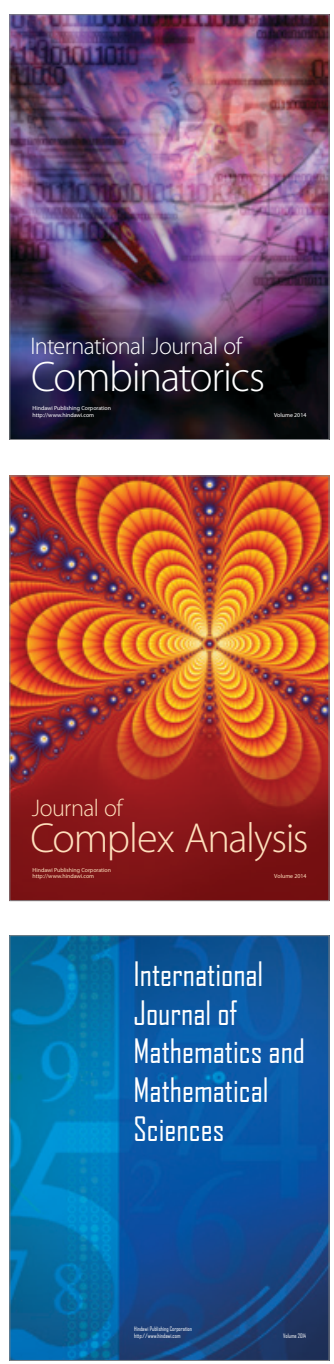
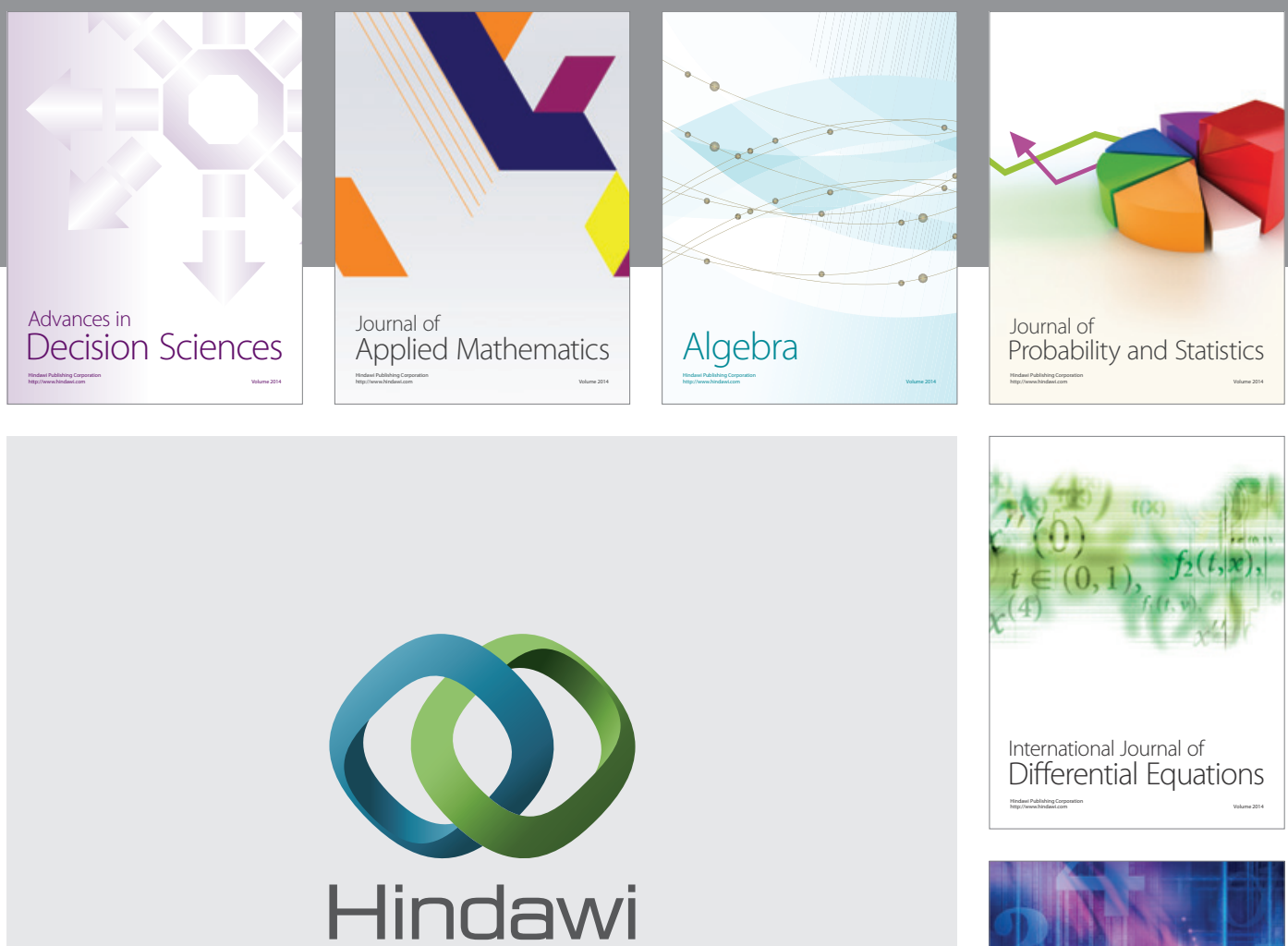

Submit your manuscripts at http://www.hindawi.com
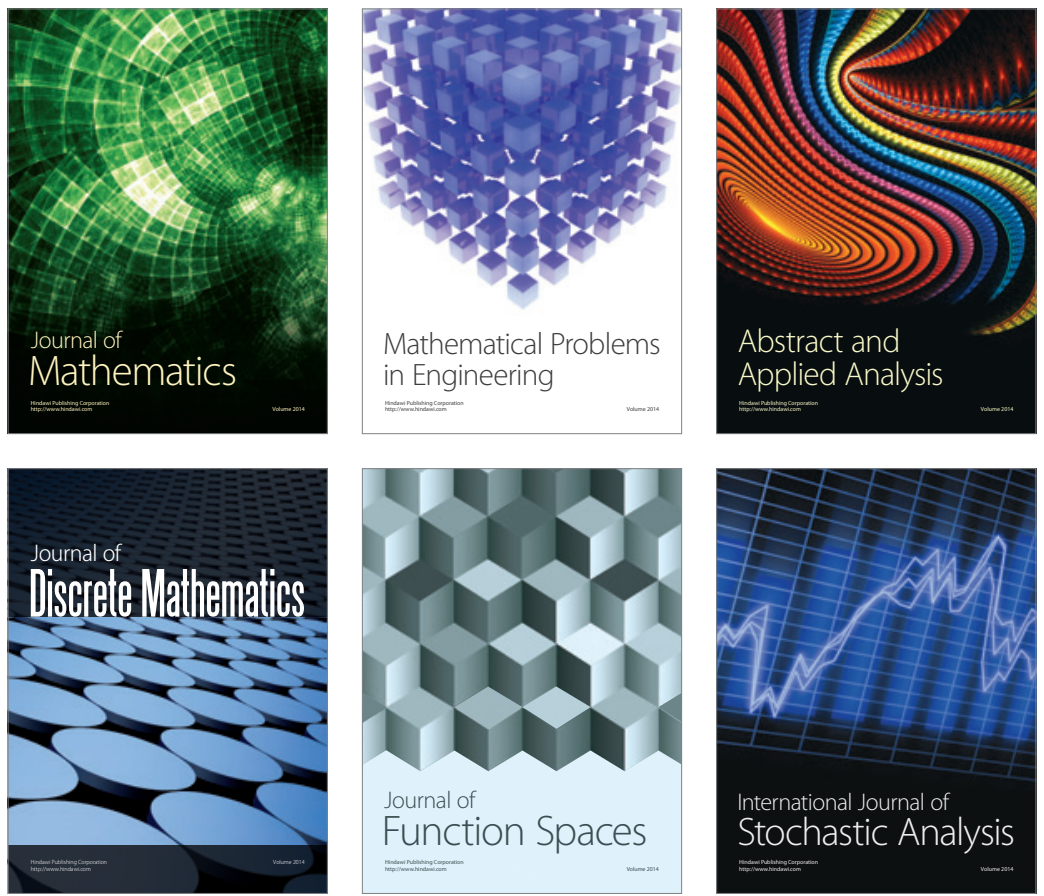

Journal of

Function Spaces

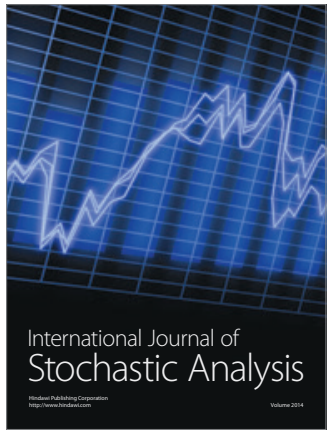

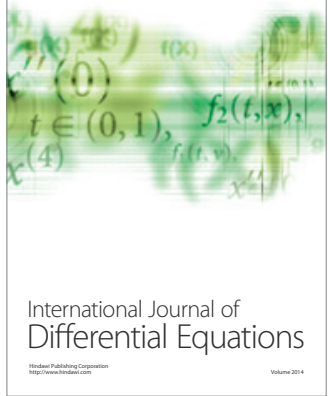
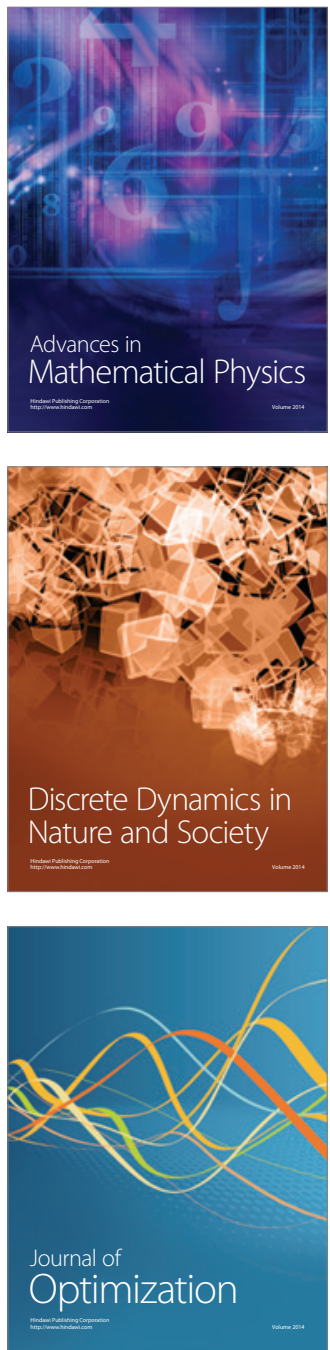